\title{
„Super-Gau“ Golf von Mexiko
}

\author{
Hans-Jörg Barth • Almut B. Heinrich
}

Erhalten: 4. Juni 2010/Akzeptiert: 6. Juni 2010/Online veröffentlicht: 26. Juni 2010

(C) Springer-Verlag 2010

\section{Einleitung}

Der GAU, der größte für das Vorsorgekonzept angenommene Ölunfall an der deutschen Nordseeküste, ist der, bei dem 15000 tÖl freigesetzt werden. Darauf sind die Vorsorgemaßnahmen abgestellt. Per definitionem kann ein GAU übertroffen werden, doch reichen dann die Vorsorgemaßnahmen nicht mehr aus; für diesen Fall wurde der Terminus „SuperGAU“ geprägt (Höpner 1991). Doch für das Szenario am Golf von Mexiko trifft selbst der Begriff „Super-Gau“ nicht mehr zu. Es existieren keine Worte mehr dafür, es herrscht nur noch Sprachlosigkeit vor der Ungeheuerlichkeit dieser selbstverschuldeten Katastrophe. Das bestätigt das folgende Interview mit PD Dr. Hans-Jörg Barth: Man muss auf die Selbstheilungskräfte der Natur vertrauen, doch die nötigen Zeiträume werden in Dekaden gemessen.

\section{Interview}

Red.: 1) Wie kann man in solch großen Tiefen bohren, ohne einen Notfallplan bei der Hand zu haben? Materialschäden können doch immer auftreten.

\section{H.-J. Barth}

Energie- und Umweltzentrum Allgäu (eza),

Burgstraße 26, 87435 Kempten, Deutschland

E-Mail: barth@eza.eu

Universität Regensburg, Physische Geographie, Universitätsstraße 31, 93040 Regensburg, Deutschland E-Mail: hjbarth@gmx.net

\section{A. B. Heinrich $(\bowtie)$}

Managing-Editor im Auftrag von Springer-Verlag,

Kirschgartenstr. 91, 69126 Heidelberg, Deutschland

E-Mail: abh.scientificjournals@googlemail.com
Barth: Es ist nach wie vor unverständlich, dass Genehmigungen für derartige Projekte erteilt werden, wenn keine ausgereiften Notfallpläne auf der Grundlage erprobter Technologien vorliegen. Wir erkennen hieran, dass ökonomische Interessen ganz klar favorisiert werden. Solange der Wachstumsimperativ nach wie vor Gegenstand allen politischen Geschehens ist, werden wir mit dieser Entscheidungslage leben müssen. Ein anderes sehr bekanntes Beispiel ist in diesem Falle die Nutzung der Kernenergie. Auch hier gibt es keine erprobten Notfallpläne und noch nicht einmal ein plausibles Entsorgungskonzept für die anfallenden Abfälle - und dennoch erleben wir derzeit weltweit eine Renaissance der Kernenergie.

Red.: 2) Wie könnte ein solcher Notfallplan aussehen?

Barth: Hier gibt es zwei Aspekte:

Zum einen das Bohrloch selbst - in diesem Falle müssten Sie die Ingenieure fragen. Es müssen Möglichkeiten geschaffen werden, das Bohrloch vor der Förderung zu stabilisieren und mit mehreren Verschlussmöglichkeiten bzw. Schleusen auszustatten. Wenn in ein U-Boot Wasser eindringt, dann lassen sich Türen verschließen, welche ein weiteres Vordringen des Wassers verhindern. Ähnlich sollte es im Bohrloch sein.

Zum Zweiten brauchen wir einen Notfallplan für das Öl, das - aus welchen Umständen auch immer - in das Meer gelangt. Da dies leider keine Seltenheit ist, müssten wir zumindest hier erprobte Techniken an der Hand haben. Leider existieren aber nur wenig effektive Lösungen, die zumeist bei widrigen Wetterbedingungen nicht einsetzbar sind.

Wir haben nicht einmal für Schiffsunfälle international gültige und verpflichtende Notfallpläne. Denken Sie an den Tankerunfall der „Prestige“ vor der spanischen Küste 2002. Statt das Schiff in das nächste Hafenbecken zu schleppen, wurde es auf den Atlantik hinaus befördert, wodurch sich der Schaden vervielfachte. 
Es ist auch verwunderlich, dass, obwohl verschiedene Öladsorbermaterialien existieren und in kürzester Zeit gefertigt werden könnten, nicht einmal unsere Bundesregierung bereit ist, ein größeres Kontingent auf Lager zu halten, um es im Falle eines Unfalles rasch und gezielt einsetzen zu können.

Red.: 3) Warum hat die Aktion „Top Kill“ nicht funktioniert? Ist der Druck zu groß? Lässt sich der Druck nicht berechnen?

Barth: Selbst die Ingenieure von BP wissen nicht, warum die Methode, das Bohrloch mit Spezialschlamm, Gummiresten und Faserabfällen, welche unter hohem Druck in das Bohrloch gespült wurden, nicht funktioniert hat. Es ist einfach sehr schwierig, in einer Tiefe von $1600 \mathrm{~m}$, bei Wassertemperaturen nahe $0{ }^{\circ} \mathrm{C}$, Strömungen zu modellieren und präzise zu agieren.

Red.: 4) Würde eine Versiegelung dem Druck standhalten?

Barth: Wenn man den Ölfluss für einige Zeit stoppen könnte, wäre man vermutlich mit einer dauerhaften Versiegelung erfolgreich.

Red.: 5) Warum kann man das schadhafte Steigrohr nicht ersetzen?

Barth: Man möchte es entfernen, um eine LMRP-Glocke (,lower marine riser package cap“) darüber anzubringen, die das Absaugen des Öls erlaubt. Um auf dem defekten Sicherheitsventil die Glocke mit einem Dichtungsring abschließen zu können, muss das Rohr entfernt werden. Dann können Dichtung und Glocke angebracht werden. Es darf kein Wasser eindringen, damit sich Methanol nach unten pumpen lässt, welches die Bildung von Gashydraten (wegen der Kälte) verhindern soll. Im Prinzip hat man dann eine Art Steigrohr bis zum Bohrschiff. (Red.: Inzwischen wurde diese Maßnahme durchgeführt, doch weiß man heute, am 6. Juni, noch nichts über das Ergebnis.)

Red.: 6) Warum dauert die Entlastungsbohrung so lange?

Barth: Diese muss mit außergewöhnlicher Präzision durchgeführt werden, da sie das Bohrloch schräg von oben kommend treffen muss. Auch hier sind die widrigen Bedingungen in der Tiefe problematisch.

Red.: 7) Lässt sich nicht abschätzen, wie groß der Ölvorrat noch ist?

Barth: Wenn Sie wissen, welchen Schaden 40000 t Rohöl vom Supertanker „Exxon Valdez“ angerichtet haben, denke ich, spielt es keine Rolle, ob der Ölvorrat der Lagerstätte nun noch 1 Mio. t oder 100 Mio. $t$ umfasst.

Red.: 8) Das verhängte Bohrverbot ist doch nichts anderes als ein schwächlicher Versuch einer Art Schadensbegrenzung. Es bewirkt nichts, verursacht nur noch mehr wirtschaftliche Verluste.

Barth: Es ist wie immer eine Maßnahme, die erst als Reaktion auf eine Katastrophe folgt. Natürlich ist ein Bohr- verbot für Tiefseeförderungen sinnvoll, da man die Technik nicht beherrscht und die Risiken unkalkulierbar sind. Eine sehr viel konsequentere Maßnahme, welche nicht nur die Symptome bekämpft, wäre eine drastische Förderung des Ausbaus erneuerbarer Energien in den USA und auch weltweit. Nur wenn wir von den fossilen Energierohstoffen unabhängiger werden, mindern wir den Druck auf wertvolle Ökosysteme. Ich erinnere nur an die katastrophalen Abbaumethoden bei der Förderung von Rohöl aus den Ölsanden in Canada.

Red.: 9) Das bestehende Horrorszenario vergrößert sich ins Unermessliche, wenn man die bevorstehende Hurrikanzeit und die drohenden Flutwellen bedenkt.

Barth: Auch ohne Hurrikans wird das Ö1 über die verschiedenen Strömungsmuster weiter verteilt werden, als wir uns vorstellen können. Hurrikans vermögen möglicherweise sogar, das Öl an der Wasseroberfläche mit Sauerstoff zu vermischen, sodass der natürliche Abbauprozess beschleunigt wird; zumindest die leicht flüchtigen (und giftigsten) Verbindungen werden hierbei reduziert.

Red.: 10) Wie wird man das Öl im Wasser handhaben können?

Barth: Mit den zur Verfügung stehenden Technologien überhaupt nicht, da die Dimensionen alles sprengen, womit wir bisher Erfahrung haben. Selbst die Ölkatastrophe am Arabischen Golf nach dem 2. Golfkrieg, als 700 km Küste verseucht wurden, wird hier klein erscheinen (Höpner 1991; Barth 2006).

Red.: 11) An die Umweltfolgen mag man gar nicht denken. Die ganze Region ist doch tot. Wie will man damit fertig werden?

Barth: Wir können nur auf die Selbstheilungskräfte der Natur vertrauen. Die Erfahrungen am Arabischen Golf in Saudi-Arabien und Kuwait lehren uns aber, dass auch bei hohen Temperaturen die Regenerationsprozesse in sogenannten schwachenergetischen Küstenabschnitten - und um die geht es hier hauptsächlich (Salzmarschen, Mangroven, usw.) - mehrere Jahrzehnte benötigen werden, und manchmal auch überhaupt keine Erholung stattfinden kann. Das bedeutet nichts Gutes für zahllose einzigartige Ökosysteme und die davon abhängigen Menschen.

Red.: 12) Auf BP kommen sicher unermessliche Schadensersatzansprüche zu?

Barth: Das ist das juristische Nachspiel, das den Ökosystemen auch nicht hilft. Selbst wenn der Konzern unter der Last der Entschädigungen zusammenbricht, wird es andere geben, die ähnliche Projekte planen und durchführen - vermutlich ebenfalls unter unkalkulierbaren Risiken. Ich betone nochmals, dass uns hier nur ein komplettes Umdenken weiterbringen kann. Wir müssen massiv am Ausbau der erneuerbaren Energien arbeiten, um die Abhängigkeit von den fossilen Energierohstoffen zu reduzieren und damit den Druck auf die Produzenten, in immer entlege- 
nere Gebiete mit Exploration und Förderung vordringen zu müssen.

Red.: 13) Was hat das Gespräch mit BP ergeben?

Barth: Dieses ist sehr enttäuschend verlaufen. BP zeigt zum gegenwärtigen Zeitpunkt kein Interesse daran, unsere Öladsorber-Technologie zu testen. Dabei ist das Verfahren geeignet, bereits unter Wasser Öl zu binden. Auch in Flachküstenabschnitten ist es einzusetzen. Die Ölschwämme könnten zu einem gewissen Grad sogar bei Hurrikans das Öl binden und eine weitere Verteilung reduzieren. Dennoch fehlt innovativen Lösungen wie dieser offenbar die professionelle Lobbyarbeit.

\section{Literatur}

Barth H-J (2006) Die Bedeutung von Krabben (Brachyura) bei der Regeneration von kohlenwasserstoffkontaminierten subtropischen Flachküsten am Beispiel des Arabisch Persischen Golfs. Umweltwiss Schadst Forsch 18(4)242-247

Höpner T (1991) Die Ölkatastrophe am Golf - Zwischenbilanz, Zustandsbeschreibung, Maßnahmen, Prognosen. Umweltwiss Schadst Forsch 3(6)354-361 